\title{
Melanoma-associated antigen A2 is overexpressed in glioma and associated with poor prognosis in glioma patients
}

\author{
Q. MENG*, G. LUO*, B. LIU*, Y. SUN, Z. YAN* \\ ${ }^{1}$ Department of Neurosurgery, Tangdu Hospital, Fourth Military Medical University, Xian 710038, China \\ ${ }^{*}$ Correspondence: $y z j 2008 \_z h o n g j u n @ 163 . c o m$ \\ ${ }^{\#}$ Contributed equally to this work.
}

Received June 25, 2017 / Accepted November 3, 2017

\begin{abstract}
Malignant glioma is the most common and aggressive primary brain tumor and the overall prognosis for glioma patients remains poor. Clarification of the molecular mechanism responsible for glioma progression is critical for the effective treatment of glioma. Melanoma antigen gene (MAGE)-A2 (MAGEA2) is a member of the MAGE-A family proteins widely studied for cancer vaccine development and identification of tumor markers. However, MAGEA2 clinical significance and biological function in glioma remain unclear, especially for the prognosis of glioma patients. This study investigates MAGEA2 expression in glioma tissue samples and its significance in predicting glioma patient prognosis. MAGEA2 protein expression in tissue samples was measured by immunohistochemistry and western blotting, and MAGEA2 mRNA expression was determined by real-time polymerase chain reaction. Our results confirmed that MAGEA2 mRNA and protein expression levels were upregulated in glioma tissues, compared with normal brain tissue. The high expression of MAGEA2 in glioma tissues significantly correlated with World Health Organization advanced grade. Univariate and multivariate analyses revealed that high MAGEA2 expression is an independent prognostic factor for glioma patient poor overall survival. The P53 mRNA expression levels were downregulated in glioma tissues compared to noncancerous brain tissue and MAGEA2 expression negatively correlated with P53 expression. Taken together, our results suggest that MAGEA2 plays an oncogenic role in glioma progression, and they provide insight into MAGEA2 application as a novel predictor of clinical outcomes and a potential glioma biomarker.
\end{abstract}

Key words: MAGEA2, glioma, overall survival, prognosis, biomarker

Glioma is the most common and aggressive primary brain tumor; it is invasive, aggressive and neurologically destructive. The World Health Organization (WHO) classifies it in four grades: pilocytic astrocytoma (grade I), diffuse astrocytoma (grade II), anaplastic astrocytoma (grade III) and glioblastoma multiforme (grade IV) [4]. Despite advances in surgical resection followed by a combination of radiotherapy or chemotherapy, glioma patient median survival remains at approximately one year [1-3] and it is therefore urgent to find new therapeutic strategies for glioma because of its high incidence and mortality. Exploration of new molecular biomarkers is important for better understanding of the carcinogenic mechanisms involved and also for development of patient-specific treatments and improvement in their prognosis.

Melanoma antigen gene (MAGE)-A proteins are members of the cancer-testis antigen gene family and are normally expressed in germline cells as immunogenic targets and differentially expressed in a variety of human cancers. MAGE-A proteins are part of the type I MAGE gene subfamily and MAGE-A expression is restricted to tumor and germinal cells [5]. Many tumor types have early MAGE-A expression which is associated with genome-wide hypo-methylation which is a frequent epigenetic modification in carcinogenesis [6]. While MAGE-A proteins have high sequence homology believed to be functionally redundant, they are widely studied for cancer vaccine development and identification of tumor markers [7-9]. MAGE-A protein biological function has been investigated over recent years and results show that they confer the following specific advantages to cancer cells; (1) MageA1 interacts with SKIP and interferes with Notch-IC regulation [10]: (2) MageA3 may regulate FGFR signaling $[11,12]$ and (3) MageA11 may regulate AR activation [13]. Further, the role of MAGEA2 is gaining more attention through evidence that it is a strong inhibitor of the p53 tumor-suppressor transcription factor 
by histone deacetylase (HDAC) 3 recruitment [14], and it can also interfere with p53 acetylation in human primary melanoma cells; thus conferring resistance to chemotherapeutic drugs which is then reversible by HDAC inhibitor drugs [14]. Although contrasting studies report the opposite correlation between MAGE-A gene expression and p53 activity $[15,16]$, the expression and significance of MAGEA2 in glioma tissue samples remains unknown; especially in the prognosis of glioma patients.

This study examined MAGEA2 expression in clinical glioma tissue samples and assessed relationship between its expression and the clinicopathologic features of glioma patients. Our results establish that expression level positively correlates with histologic staging and that MAGEA2 overexpression has an important role in glioma grading. It can also indicate poor clinical prognosis. Moreover, the data suggests that MAGEA2 is an independent prognostic factor of glioma patients and it can be a potential therapeutic target in glioma.

\section{Patients and methods}

Patients and tissue specimens. 120 paraffin-embedded glioma tissue specimens were retrieved from the Department of Neurosurgery, Tangdu Hospital, Fourth Military Medical University from May 2007 to June 2016. The patients did not receive any radiotherapy or chemotherapy before surgery. The histologic grade of the tumors was examined by two independent pathologists according to the WHO classification system. Overall survival (OS) was defined as the time from the date of diagnosis to the date of death, or the date of the last follow-up if death did not occur. We also obtained 12 non-tumorous brain tissue specimens and 78 fresh glioma tissue specimens from the Department of Neurosurgery, Tangdu Hospital, Fourth Military Medical University from May 2007 to June 2016. The normal brain tissue specimens were excised from patients with cerebral trauma or cerebral hemorrhage. All procedures were carried out in accordance with the Internal Review and the Ethics Board of the above hospital, its Ethics Committee approved the study and informed consent was obtained from all participating patients. The patients' clinico-pathologic characteristics are detailed in Table 1.

Immunohistochemical staining of tissue specimens. Immunohistochemistry analyzed MAGEA2 expression. In brief, paraffin-embedded specimens were cut into 4 $\mu \mathrm{m}$ sections. After baking at $65^{\circ} \mathrm{C}$ for 1 hour, the sections were deparaffinized and rehydrated. Endogenous peroxidase activity was blocked by $3 \%$ hydrogen peroxide for 10 minutes at room temperature, followed by incubation with $1 \%$ bovine serum albumin to block nonspecific binding. Rabbit anti-MAGEA2 (1:100 dilution; Santa Cruz Biotechnology) was incubated with the sections overnight at $4^{\circ} \mathrm{C}$. Normal goat serum was used as negative controls. After washing, the tissue sections were incubated with biotinylated anti-rabbit secondary antibody for $30 \mathrm{~min}$ at room tempera- ture and then counterstained with hematoxylin, dehydrated and coverslipped.

Scoring of staining. The tissue specimen immunostaining degree was scored according to the proportion of positively stained tumor cells and the staining intensity. This scoring was performed by two independent investigators who were 'blinded' to the sample histopathologic features and patient data. The averaged scoring of MAGEA2 expression was calculated based on the scores given by the two independent investigators, and the proportion of positive stained tumor cells was graded as follows: 0 (no positive tumor cells), $1(<10 \%$ positive tumor cells), 2 ( $10-50 \%$ positive tumor cells) and 3 ( $>50 \%$ positive tumor cells). The intensity of staining was then graded: 0 (no staining), 1 (weak staining, light yellow), 2 (moderate staining, yellowish brown) and 3 (strong staining, brown). The stage of tumor severity was evaluated by the staining index (SI), SI = staining intensity $\times$ proportion of positively stained tumor cells. High and low MAGEA2 expression was defined by the measure of heterogeneity with the log-rank test statistics for OS; 0-3 indicates low expression and 4-9 reveals high expression. MAGEA2 tissue specimen expression was quantitatively analyzed by the AxioVision Rel. 4.6 computerized image system equipped with automatic measurement program (Carl Zeiss, Oberkochen, Germany). The stained tissues were observed under 200x magnification and six representative staining fields of each section were analyzed to evaluate the mean optical density (MOD).

RNA isolation and quantitative RT-PCR. Total RNA was extracted from brains by RNA isolation kit and then reversely transcribed into cDNA using miScript II RT Kit (Thermo Fisher Scientific Inc., Waltham, MA, USA), according to the manufacturer's instructions. cDNA was used as a template in PCR reactions with gene-specific primer pairs. Realtime PCR was performed by miScript SYBR Green PCR Kit (Thermo Fisher Scientific Inc., Waltham, MA, USA) with $\beta$-actin as endogenous control. Target gene expression fold changes were determined by $\Delta \Delta \mathrm{Ct}$, and relative expression of these genes was calculated by the $2^{-\Delta \mathrm{CT}}$ method and normalized to $\beta$-actin.

Western blotting. Tissue samples were homogenized in ice-cold RIPA lysis buffer with protease inhibitor cocktail kit (Sigma-Aldrich, St. Louis, MO). Protein samples were subjected to SDS-PAGE and then transferred to PVDF membrane and incubated overnight at $4{ }^{\circ} \mathrm{C}$ with primary antibodies ( $\beta$-actin: Santa Cruz, 1:500 and MAGEA2: Abcam, 1:1000). After washing with TBST, the membranes were incubated at $37^{\circ} \mathrm{C}$ for 1 hour with horseradish peroxidase (HRP)-conjugated secondary antibodies (Thermofisher Scientific, USA). Immunoreactive proteins were visualized with enhanced chemiluminescence captured by BIORAD Imaging Systems (BIORAD, CA, USA). Equal loading was ensured by detection of $\beta$-actin.

Statistical analysis. Data was analyzed by SPSS17.0 statistical package (SPSS Inc., Chicago, IL, USA), and associations 
Table 1. Relationship between MAGEA2 levels and clinicopathologic characteristics in 120 glioma specimens.

\begin{tabular}{|c|c|c|c|c|c|}
\hline \multirow[b]{2}{*}{ Variables } & \multirow{2}{*}{$\begin{array}{c}\text { Number } \\
\text { of cases } \\
(\mathrm{N}=120)\end{array}$} & \multicolumn{2}{|c|}{ MAGEA2 expression } & \multirow[b]{2}{*}{$\chi^{2}$} & \multirow[b]{2}{*}{ p-value } \\
\hline & & $\begin{array}{c}\text { Low } \\
(n=49)\end{array}$ & $\begin{array}{c}\text { High } \\
(n=71)\end{array}$ & & \\
\hline Age (years) & & & & 0.0026 & 0.8943 \\
\hline$\leq 50$ & 58 & 23 & 35 & & \\
\hline$>50$ & 62 & 26 & 36 & & \\
\hline Gender & & & & 3.219 & 0.0621 \\
\hline Female & 59 & 26 & 33 & & \\
\hline Male & 61 & 23 & 38 & & \\
\hline Tumor size $(\mathrm{cm})$ & & & & 37.73 & $<0.0001$ \\
\hline$\leq 5$ & 51 & 31 & 20 & & \\
\hline$>5$ & 69 & 18 & 51 & & \\
\hline Karnofsky score & & & & 31.36 & $<0.0001$ \\
\hline$\leq 90$ & 75 & 16 & 59 & & \\
\hline$>90$ & 45 & 33 & 12 & & \\
\hline WHO grade & & & & 26.73 & $<0.0001$ \\
\hline $\mathrm{I}+\mathrm{II}$ & 77 & 32 & 45 & & \\
\hline III+IV & 43 & 17 & 26 & & \\
\hline
\end{tabular}

Note: achi-square test.

Abbreviations: I, WHO grade I pilocytic astrocytoma; II, WHO grade II diffuse astrocytoma; III, WHO grade III anaplastic astrocytoma; IV, WHO grade IV glioblastoma multiforme; WHO, World Health Organization; MAGEA2, Melanoma-associated antigen A2.

of clinical-pathologic parameters with MAGEA2 expression were determined by chi-square test. The OS was evaluated by Kaplan-Meier analysis and the log-rank test compared differences between groups. Univariate Cox regression analysis then evaluated the prognostic significance of clinical and pathologic characteristics. All statistical tests were two-tailed, with errors standard deviation (SD) of averaged results and p-values $<0.05$ considered statistically significant.

\section{Results}

MAGEA2 expression is correlated with clinicopathologic features of patients with glioma. Table 1 highlights the relationship between MAGEA2 expression levels and patient glioma tumor clinical-pathologic features. MAGEA2 expression in 120 glioma tissue specimens was evaluated by IHC. These came from 120 glioma patients; 59 women $(49.17 \%)$ and 61 men $(50.83 \%) 16$ to 78 years old with median age of 49.6 years. According to 2007 WHO classification, these consisted of 28 patients with grade I astrocytomas, 49 with grade II, 21 with grade III and 22 grade IV. We observed that $59.17 \%$ of tissue samples (71/120) exhibited high MAGEA2 expression and $27.22 \%(49 / 180)$ had low expression. The proportion of tumors with $\leq 5$ diameter was $42.5 \%(51 / 120)$ and $>5$ was $57.5 \%(69 / 120)$. This latter $57.5 \%$ comprised $71.83 \%(51 / 71)$ in the high MAGEA2 expression group and $36.73 \%$ (18/49) in the low MAGEA2 expression group. KPS was also evaluated for all the patients before surgery. High MAGEA2 expression levels were significantly associated with large tumor size $(>5 \mathrm{~cm} ; \mathrm{p}<0.0001)$, low KPS score $(<90 ; \mathrm{p}<0.0001)$ and high WHO grade $(\mathrm{p}<0.0001)$. However, we observed no significant correlation between MAGEA2 expression levels and age or gender.

Correlation between MAGEA2 expression and glioma grade. To examine whether MAGEA2 expression level was associated with glioma grade, IHC was performed to determine MAGEA2 expression in non-tumorous brain tissues and glioma tissue specimens. The images of MAGEA2 staining obtained from glioma patients and non-tumorous brain tissue are highlighted in Figure 1A. MAGEA2 staining gradually increased as tumor grade rose. We compared MAGEA2 staining mean optical density (MOD) in 12 non-tumorous brain tissues and all grades of the glioma

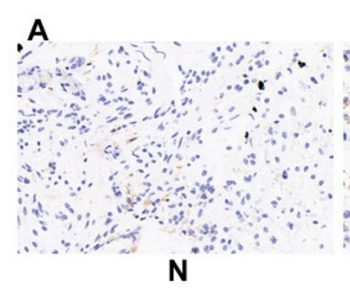

B



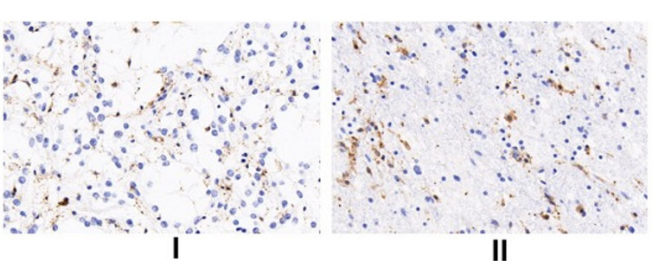

C

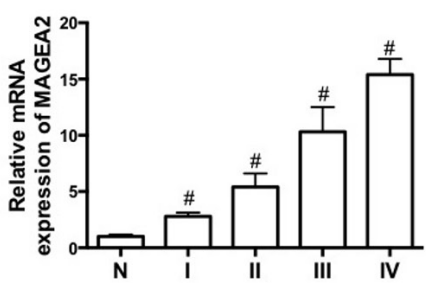



D

MAGEA2

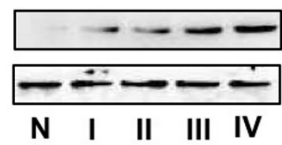

Figure 1. MAGEA2 expression is increased in glioma tissues. (A) Immunostaining of MAGEA2 in paraffin-embedded specimens, including 12 nontumor brain tissues and 120 glioma tissues with WHO grade I to IV. (magnification $\times 200$ ). (B) Comparative quantification of the mean optical density (MOD) of MAGEA2 staining in different specimens. (C) Relative mRNA expression of MAGEA2 in 78 tissue samples (12 non-tumor brain tissues, 13 GI, 18 GII, 16 GIII and 19 GIV glioma tissue samples). (D) Protein expression of MAGEA2 in tissue samples. ${ }^{*} \mathbf{p}<0.05$ vs control. 
tissue. The MOD of MAGEA2 staining increased stepwise from lower to higher grade $(\mathrm{p}<0.05$; Figure $1 \mathrm{~B})$. MAGEA2 mRNA expression was also determined by qRT-PCR analysis in 78 glioma tissue specimens and 12 non-tumorous brain tissue specimens. MAGEA2 mRNA and protein levels were markedly increased in all grades of glioma tissue specimens $(\mathrm{p}<0.05$; Figure $1 \mathrm{C}$ and D) compared to the non-tumorous brain tissue. Moreover, the MAGEA2 mRNA and protein expression increased from lower grade to higher grade in all gliomas $(\mathrm{p}<0.05$; Figure $1 \mathrm{C}$ and $\mathrm{D})$. These results suggest MAGEA2's important role in glioma development.

Positive expression of MAGEA2 is correlated with poor prognosis of glioma patients. MAGEA2 was divided into high and low expression groups to assess the correlation between protein expression and glioma patient prognosis. The categorization was defined on the basis of positively stained tumor cells and intensity of staining. Kaplan-Meier survival analysis was performed to investigate the prognostic significance of MAGEA2 levels in patients with glioma. We showed that patients with high MAGEA2 levels had a significantly shorter OS than those with low MAGEA2 levels $(\mathrm{p}<0.0001$; Figure 2$)$. In addition, the median survival time of patients whose tumors had high MAGEA2 expression levels was only 22 months (hazard ratio $[\mathrm{HR}]=0.3762$; 95\% confidence interval [CI]: 0.2713-0.5237), whereas the median survival time of those with low expression level was 46 months ( $\mathrm{HR}=2.238$; 95\% CI: $1.583-2.904)$. Moreover, the cumulative 5-year survival rate was $40.81 \%(20 / 49)$ in the low MAGEA2 expression group, whereas it was only $7.04 \%(5 / 71)$ in the high expression group. The relative risk of prognostic parameters was also determined using univariate and multivariate analyses for the 120 glioma patients (Table 2). Results indicated that the MAGEA2 expression level, tumor size and WHO grade were independent prognostic factors for poor OS in glioma patients, and that MAGEA2 protein over-expression correlated with poor prognosis in glioma clinical patients.

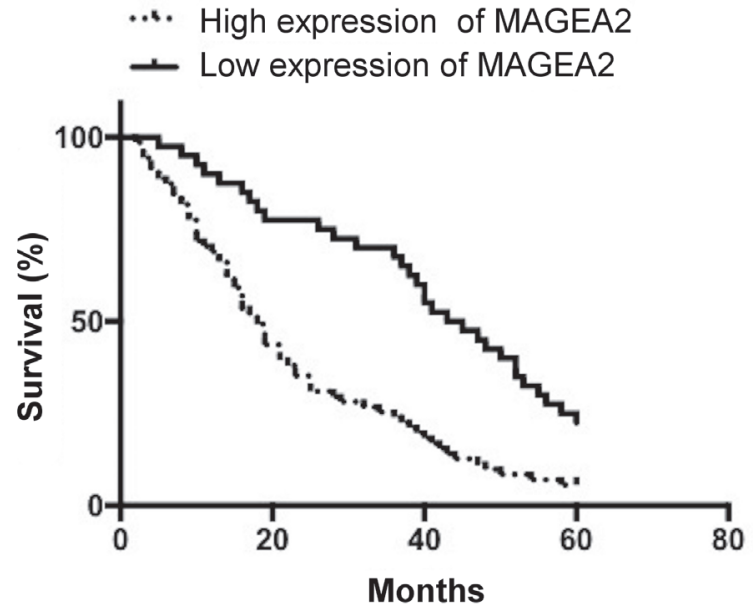

Figure 2. Kaplan-Meier curves with log-rank test for patients with low MAGEA2 expression (bold line) versus high MAGEA2 expression tumors (dotted line). High MAGEA2 expression was markedly associated with a reduced overall survival in glioma patients $(\mathrm{p}<0.0001)$.

MAGEA2 expression is negatively correlated with P53 expression. The next step evaluated the possible relationship between MAGEA2 and P53 expression. We compared MAGEA2 mRNA expression in 12 non-tumorous brain tissues and all grades of studied glioma tissue. The results showed that the levels of P53 mRNA were markedly decreased in all grades of glioma tissue specimens compared with non-tumorous brain tissues $(\mathrm{p}<0.05$; Figure $3 \mathrm{~A})$. Moreover, the P53 mRNA expression decreased from lower grade to higher grade in all gliomas $(\mathrm{p}<0.05$; Figure $3 \mathrm{~A})$. MAGEA2 expression was inversely correlated with the P53 expression $\left(\mathrm{R}^{2}=0.6428\right.$; Figure $\left.3 \mathrm{~B}\right)$. These results indicated that the MAGEA2 oncogenic role in glioma progression may be associated with P53.downregulation.

Table 2. Univariate and multivariate analyses of prognostic parameters for survival in glioma patients.

\begin{tabular}{lccccc}
\hline Variables & HR & $\begin{array}{c}\text { Univariate analysis } \\
\mathbf{9 5 \%} \text { CI }\end{array}$ & p-value & HR & $\begin{array}{c}\text { Multivariate analysis } \\
\mathbf{9 5 \%} \text { CI }\end{array}$ \\
\hline $\begin{array}{l}\text { Age (years) } \\
\leq 50 \text { vs }>50\end{array}$ & 0.858 & $0.509-3.567$ & 0.561 & & \\
$\begin{array}{l}\text { Gender } \\
\text { Female vs Male }\end{array}$ & 0.914 & $0.659-3.814$ & 0.603 & & \\
$\begin{array}{l}\text { Tumor size (cm) } \\
\leq 5 \text { vs }>5\end{array}$ & 0.582 & $0.362-0.891$ & $<0.001^{*}$ & 0.613 & $0.389-0.971$ \\
$\begin{array}{l}\text { MAGEA2 level } \\
\text { Low vs high }\end{array}$ & & & & $1.582-3.894$ \\
$\begin{array}{l}\text { WHO grade } \\
\text { I+II vs III+IV }\end{array}$ & 2.345 & $1.489-3.672$ & $<0.0001^{*}$ & 2.473 & $<0.001^{*}$ \\
\hline
\end{tabular}

Notes: ${ }^{a} \log$-rank test. ${ }^{*} \mathrm{p}<0.05$

Abbreviations: I, WHO grade I pilocytic astrocytoma; II, WHO grade II diffuse astrocytoma; III, WHO grade III anaplastic astrocytoma; IV, WHO grade IV glioblastoma multiforme; CI, confidence interval; HR, hazard ratio; WHO, World Health Organization 
A

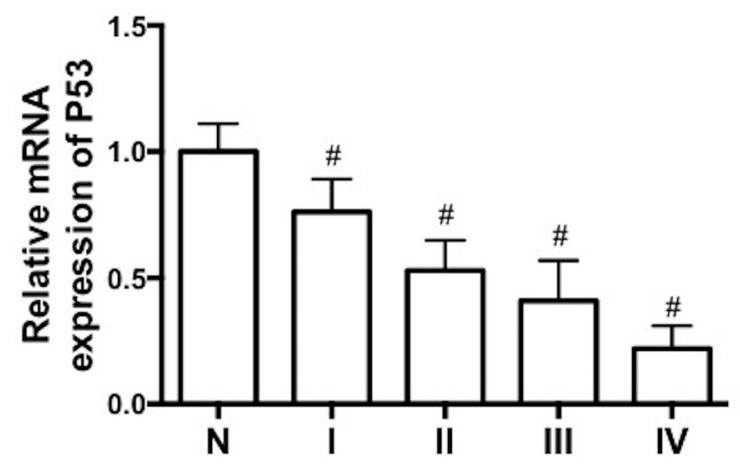

B

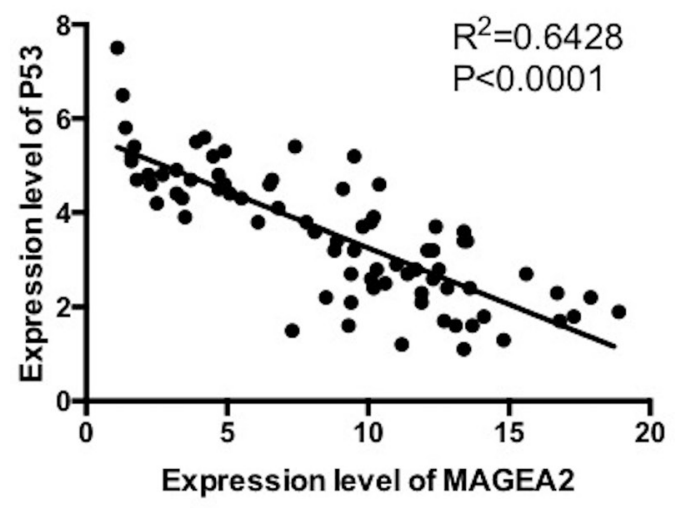

Figure 3. MAGEA2 expression is negatively correlated with P53 expression in glioma tissues. (A) Relative mRNA expression of P53 in 78 tissue samples (12 non-tumor brain tissues, 13 GI, 18 GII, 16 GIII and 19 GIV glioma tissue samples). (B) Correlation between MAGEA2 and P53 expression in glioma tissues. ${ }^{\#} \mathrm{p}<0.05$, vs control.

\section{Discussion}

Malignant glioma is the most common and aggressive primary brain tumor and the overall prognosis for glioma patients remains poor $[17,18]$. Clarification of the molecular mechanism responsible for glioma progression is critical for effective treatment of glioma [19]. The main finding in the current study is that the progression of human glioma is associated with upregulation of MAGEA2 expression. We found that high MAGEA2 expression levels were notably associated with large tumor size $(>5 \mathrm{~cm} ; \mathrm{p}<0.0001)$, low KPS score $(<90 ; \mathrm{p}<0.0001)$ and high WHO grade $(\mathrm{p}<0.0001)$. Moreover, IHC and qRT-PCR results showed that MAGEA2 expression in glioma tissue samples was increased compared with non-tumorous brain tissues. The MAGEA2 staining score increased stepwise from lower to higher grade glioma tissues, and MAGEA2 mRNA and protein expression levels also increased from lower to higher grades. The prognostic significance of MAGEA2 levels in glioma patients was evaluated by Kaplan-Meier survival analysis, and results confirmed that patients with high MAGEA2 levels had a remarkably shorter OS than those with low MAGEA2 levels $(\mathrm{p}<0.0001)$. In addition, the cumulative 5-year survival rate was significantly higher in the low MAGEA2 expression group than in the high expression group. The results demonstrate the expression format and prognostic importance of MAGEA2 expression in human glioma tissue samples, and these suggest that MAGEA2 acts as an independent prognostic factor for glioma patient overall survival.

Accumulating evidence indicates that MAGEA2 expression level is associated with cancer progression and that MAGEA2 over-expression's prognostic significance makes it a potential therapeutic target for patients with lung cancer [20]. MAGEA2 also promotes breast cancer progression by regulating Akt and Erk1/2 pathways [21] and MAGEA proteins may suppress the 53 transcriptional program during tumor development [16]. Enhanced MAGEA2 protein levels correlate with impaired acetylation of both $\mathrm{p} 53$ and histones surrounding the p53-binding sites [14]. Further, MAGEA2 restrains cellular senescence by targeting the function of PMLIV/p53 axis at the PML-NBs [22]. MAGEA2 is also differentially expressed in head and neck squamous cell carcinoma and it functions, in part, through the p53 pathway by increasing cellular proliferation and abrogating cell cycle arrest [23]. In particular, it has been shown that induced loss of MAGEA2 expression correlates with increased caspase 3/7 activity, BCL2/BAX ratio and TUNEL signal [24].

Our study reveals that MAGEA2 expression is negatively correlated with the P53 expression down-regulated in glioma tissues. Together with previous reports, our findings suggest that the interaction between MAGEA2 and P53 is involved in MAGEA2's oncogenic role in glioma. However, further study is required to clarify the mechanism underlying MAGEA2's oncogenic and prognostic significance and its directional interaction with P53. Finally, these observations and our results confirm that MAGEA2 can not only act as a potential predictive biomarker for the prognosis of gliomas but it can also be a potential therapy target.

\section{References}

[1] SIEGEL RL, MILLER KD, JEMAL A. Cancer statistics, 2016. CA Cancer J Clin 2016; 66: 7-30. https://doi.org/10.3322/ caac. 21332

[2] SHERMAN JH, HOES K, MARCUS J, KOMOTAR RJ, BRENNAN CW et al. Neurosurgery for brain tumors: update on recent technical advances. Curr Neurol Neurosci Rep 2011; 11: 313-319. https://doi.org/10.1007/s11910-011-0188-9 
[3] WEN PY, KESARI S. Malignant gliomas in adults. N Engl J Med 2008; 359: 492-507. https://doi.org/10.1056/NEJMra0708126

[4] LOUIS DN, OHGAKI H, WIESTLER OD, CAVENEE WK, BURGER PC et al. The 2007 WHO classification of tumours of the central nervous system. Acta Neuropathol 2007; 114: 97-109. https://doi.org/10.1007/s00401-007-0243-4

[5] CHOMEZ P, DE BACKER O, BERTRAND M, DE PLAEN E, BOON T et al. An overview of the MAGE gene family with the identification of all human members of the family. Cancer Res 2001; 61: 5544-5551.

[6] DE SMET C, LURQUIN C, LETHE B, MARTELANGE V, BOON T. DNA methylation is the primary silencing mechanism for a set of germ line- and tumor-specific genes with a CpG-rich promoter. Mol Cell Biol 1999; 19: 7327-7335.

[7] SANG M, WANG L, DING C, ZHOU X, WANG B et al. Melanoma-associated antigen genes - an update. Cancer Lett 2011; 302: 85-90. https://doi.org/10.1016/j.canlet.2010.10.021

[8] CABALlERO OL, CHEN YT. Cancer/testis (CT) antigens: potential targets for immunotherapy. Cancer Sci 2009; 100: 2014-2021. https://doi.org/10.1111/j.13497006.2009.01303.x

[9] KRUIT WH, VAN OJIK HH, BRICHARD VG, ESCUDIER B, DORVAL $T$ et al. Phase $1 / 2$ study of subcutaneous and intradermal immunization with a recombinant MAGE-3 protein in patients with detectable metastatic melanoma. Int J Cancer 2005; 117: 596-604. https://doi.org/10.1002/ ijc. 21264

[10] LADURON S, DEPLUS R, ZHOU S, KHOLMANSKIKH O, GODELAINE D et al. MAGE-A1 interacts with adaptor SKIP and the deacetylase HDAC1 to repress transcription. Nucleic Acids Res 2004; 32: 4340-50. https://doi. org/10.1093/nar/gkh735

[11] ZHU X, ASA SL, EZZAT S. Fibroblast growth factor 2 and estrogen control the balance of histone 3 modifications targeting MAGE-A3 in pituitary neoplasia. Clin Cancer Res 2008; 14: 1984-1996. https://doi.org/10.1158/1078-0432. CCR-07-2003

[12] KONDO T, ZHU X, ASA SL, EZZAT S. The cancer/testis antigen melanoma-associated antigen-A3/A6 is a novel target of fibroblast growth factor receptor 2-IIIb through histone H3 modifications in thyroid cancer. Clin Cancer Res 2007; 13: 4713-4720. https://doi.org/10.1158/1078-0432.CCR-070618

[13] ASKEW EB, BAI S, HNAT AT, MINGES JT, WILSON EM. Melanoma antigen gene protein-A11 (MAGE-11) F-box links the androgen receptor $\mathrm{NH} 2$-terminal transactivation domain to p160 coactivators. J Biol Chem 2009; 284: $34793-$ 34808. https://doi.org/10.1074/jbc.M109.065979
[14] MONTE M, SIMONATTO M, PECHE LY, BUBLIK DR, GOBESSI $S$ et al. MAGE-A tumor antigens target p53 transactivation function through histone deacetylase recruitment and confer resistance to chemotherapeutic agents. Proc Natl Acad Sci U S A 2006; 103: 11160-11165. https://doi. org/10.1073/pnas.0510834103

[15] YANG B, O'HERRIN SM, WU J, REAGAN-SHAW S, MA Y et al. MAGE-A, mMage-b, and MAGE-C proteins form complexes with KAP1 and suppress p53-dependent apoptosis in MAGE-positive cell lines. Cancer Res 2007; 67: 9954-9962. https://doi.org/10.1158/0008-5472.CAN-07-1478

[16] MARCAR L, MACLAINE NJ, HUPP TR, MEEK DW. MageA cancer/testis antigens inhibit $\mathrm{p} 53$ function by blocking its interaction with chromatin. Cancer Res 2010; 70: $10362-$ 10370. https://doi.org/10.1158/0008-5472.CAN-10-1341

[17] VAN DEN BENT MJ, BROMBERG JE. Neuro-oncology: The many challenges of treating elderly glioblastoma patients. Nat Rev Neurol 2015; 11: 374-375. https://doi.org/10.1038/ nrneurol.2015.82

[18] YANG P, WANG Y, PENG X, YOU G, ZHANG W et al. Management and survival rates in patients with glioma in China (2004-2010): a retrospective study from a singleinstitution. J Neurooncol 2013; 113: 259-266. https://doi. org/10.1007/s11060-013-1103-9

[19] LOUIS DN. Molecular pathology of malignant gliomas. Annu Rev Pathol 2006; 1: 97-117. https://doi.org/10.1146/ annurev.pathol.1.110304.100043

[20] UJIIE H, KATO T, LEE D, HU HP, FUJINO K et al. Overexpression of MAGEA2 has a prognostic significance and is a potential therapeutic target for patients with lung cancer. Int J Oncol 2017; 50: 2154-2170. https://doi.org/10.3892/ ijo.2017.3984

[21] PARK S, SUNG Y, JEONG J, CHOI M, LEE J et al. hMAGEA2 promotes progression of breast cancer by regulating Akt and Erk1/2 pathways. Oncotarget 2017; 8: 37115-37127. https://doi.org/10.18632/oncotarget.16184

[22] PECHE LY, SCOLZ M, LADELFA MF, MONTE M, SCHNEIDER C. MageA2 restrains cellular senescence by targeting the function of PMLIV/p53 axis at the PML-NBs. Cell Death Differ 2012; 19: 926-936. https://doi.org/10.1038/ cdd.2011.173

[23] GLAZER CA, SMITH IM, BHAN S, SUN W, CHANG SS et al. The role of MAGEA2 in head and neck cancer. Arch Otolaryngol Head Neck Surg 2011; 137: 286-293. https://doi. org/10.1001/archoto.2011.2

[24] ZENG L, KANG C, DI C, FEE BE, RIVAS M et al. The adherens junction-associated protein 1 is a negative transcriptional regulator of MAGEA2, which potentiates temozolomide-induced apoptosis in GBM. Int J Oncol 2014; 44: 1243-1251. https://doi.org/10.3892/ijo.2014.2277 